\title{
The Importance of Fuzzy Preference in Course Assignment Problem
}

\author{
Artur Gorka $^{1}$ and Phantipa Thipwiwatpotjana ${ }^{2}$ \\ ${ }^{1}$ Department of Mathematics, Erskine College, Due West, SC 29639, USA \\ ${ }^{2}$ Department of Mathematics and Computer Science, Faculty of Science, Chulalongkorn University, Bangkok 10330, Thailand \\ Correspondence should be addressed to Phantipa Thipwiwatpotjana; phantipa.t@chula.ac.th
}

Received 25 March 2015; Revised 16 June 2015; Accepted 18 June 2015

Academic Editor: Guiming Luo

Copyright ( 2015 A. Gorka and P. Thipwiwatpotjana. This is an open access article distributed under the Creative Commons Attribution License, which permits unrestricted use, distribution, and reproduction in any medium, provided the original work is properly cited.

\begin{abstract}
This paper points out the importance of fuzzy preference by using a teaching course assignment problem as a case study. The model with fuzzy teaching preference provides a more satisfactory solution to a course assignment problem than assigning arbitrary weights. A method for improving a fuzzy membership function by using sensitivity analysis is devised. The method with fuzzy preferences is compared with a model using weighted probabilities.
\end{abstract}

\section{Introduction}

One of the important inputs in course assignment problem for assigning teaching courses to instructors is the instructor preferences. Under the intuitive idea that an instructor would prefer to teach her specialty subjects, there is no need to include teacher's professional knowledge under consideration, as it creates unnecessary information and complication. Therefore one reasonable way instructors could give their preferences on their preferred subject is to set up a ranking system. The ranking is the same for all instructors, although the real feeling of each rank for each individual may be slightly different. The preference is a fuzzy concept.

There have been some papers showing use of fuzzy preference in course assignment problem. A fuzzy example of membership for each preference description was presented in [1] and then [2] applied this fuzzy preference in the course assignment problem with interval workload data. Duan et al. [3] used fuzzy synthetic decision making in genetic algorithm. There, the authors applied discretization degree to measure each combination of university curricula. This degree, which was not explicitly shown how to find it, has fuzzy sense depending on a decision maker. Ismayilova et al. [4] considered preferences of instructors and administration in their model. They applied Analytic Hierarchy and Network
Processes for weighing different and conflicting objectives. However, they used numbering of preference levels as 1,2 , 3 , and so on. The results of our research show that these preference levels may not provide the most efficient result to all course assignment problems, even though their result optimized their model. Some other articles, for example, $[5,6]$, used heuristic/metaheuristic together with fuzzy idea to generate a course schedule.

Unfortunately, none of these articles explain how to obtain such membership functions and how the authors know that their chosen preferences make a significant difference in their solutions. This is what we show in this paper.

Teaching Preference as a Fuzzy Concept. The ranks of teaching preference are usually given as $1,2,3$, and so forth. The decision maker assigns weights, or fuzzy memberships, or probabilities to those ranks. In Section 2, we first try to critique (as a decision maker) how difficult it is to weight these ranks. It is nearly impossible to know in advance an ideal weight that a decision maker would use for finding a "reasonable" assignment for teaching loads. In Section 3 we show a simple mixed-integer linear programming model using model weights. The weights are scaled to compare this model with fuzzy membership function model. The results show that weighting by fuzzy preference provides 
a better assignment. The difficult question is what should be its prototype membership function. Section 5 makes an effort to answer this question.

Teaching preference is a good example that you cannot just use probability as shown in Klir [7]. There is a degree of uncertainty there that calls for fuzzy weights. A decision maker may try to push subjects to teachers who have the most preference on those subjects. This may result in some teachers having more preferable courses than some others. Then more work needs to be done to make it fair to all teachers. If a decision maker interprets teaching preference ranks as a probability, the result would depend on fuzzy weights that need to be used for each rank of preference. We explain this in more detail in Section 6.

Meaning of Optimal Solution. This research is not trying to come up with a universal teaching assignment problem solution for all situations or all institutions and departments. It is impossible to do so. Each institution has its own unique conditions. Some may have conflicts among some instructors; some courses may have been designed by/for some individuals. Users need to add these restrictions to the model themselves. Our objective is to show the importance of fuzzy preference and that it needs to be adjusted to particular situation.

What we will do here is to verify that instructor preference definitely needs to be captured as fuzzy information. We will use the given data and standard constraints presented in the next section to show that weights representing the teaching preference need to be changed to fuzzy membership function for the preference.

To make the design of the problem easy to follow, we use a simple mixed-integer linear programming model. Consider having a duty to match instructors with subjects in order to make everyone happy using preference inputs and still satisfy restrictions at the same time.

An optimal solution to this problem is given in a sense that the number of nonpreferred subjects assigned to instructors and deviations from the real and expected workloads would be as small as possible while the total preferences of all instructors would be maximized.

\section{Instructors Preference}

The data used in this paper were generated by the Department of Mathematics and Computer Science, Faculty of Science, Chulalongkorn University, Thailand. The process starts with collecting instructors' preferences using ranks from 1 to 6 for each subject that matches their preference. The rank descriptions are shown in Table 1.

2.1. Choosing the Weights of Ranks. The teaching preference is a subjective feeling. Even though it carries practical information for each instructor, for example, specialty, years of experience, and research area, one can view the teaching preference as fuzzy information. The "weight of rank" column in Table 1 is one of many reasonable sets of weights of ranks. A researcher can come up with these numbers by asking
TABLE 1: Teaching preference rank description.

\begin{tabular}{llcc}
\hline Rank & Rank description & $\begin{array}{c}\text { Weight } \\
\text { of rank }\end{array}$ & $\begin{array}{c}\text { Fuzzy } \\
\text { number } \\
\text { of preference }\end{array}$ \\
\hline 1 & Prefers most to teach the subject & 10,000 & 1 \\
2 & Prefers to teach the subject & 5,000 & 0.8 \\
3 & OK to teach the subject & 500 & 0.7 \\
4 & Able to teach the subject & 100 & 0.6 \\
5 & $\begin{array}{l}\text { Able but does not want to teach } \\
\text { the subject }\end{array}$ & 20 & 0.3 \\
6 & Cannot teach the subject & 0 & 0 \\
\hline
\end{tabular}

instructors to provide their own sets of values of ranks within a range of 0 to 10,000 , for example, or using weights suitable for the type of work from his/her previous experience. A question raised here could be "what is meant by a 'reasonable' set of weights of ranks?."

An answer to this question is a subjective matter; however, we provide an idea of how a decision maker would decide the values of weights in the following way. Let us think of a situation where we want to weight these ranks so that an instructor who chooses rank 1 has a bigger chance to teach the subject he/she prefers the most. A decision maker would choose a very high number to value rank 1 and use a very small number for rank 6, so that an instructor has a smaller chance to be assigned a subject she does not want to teach when running the problem of maximizing the overall values of ranks. The actual formulation of optimization of the overall values of ranks and managing both the number of nonpreferred subjects and deviations from the real and expected workloads is explained in Section 3.

2.2. Choosing the Fuzzy Preference. The last column of Table 1 is a membership of the fuzzy number of preference that is provided by a decision maker. These numbers have been selected based on previous work with the teaching assignment problem in [1]. The choice of these numbers is somewhat arbitrary and is based on previous experience but can be inferred from the probabilities calculated from instructors' preferences, as shown in Section 6.

An optimal assignment obtained by using the particular fuzzy preference in the model satisfies the following objectives:

(a) Maximize the number of subjects assigned with rank 1 and minimize the number of subjects assigned with ranks 6 and 5, consecutively.

(b) Maximize the overall preferences.

(c) Minimize the total over/under workload from the requested amounts.

2.3. Comparison of Weights and Fuzzy Preference. At this stage we have two alternatives for capturing the fuzzy information: the weights or fuzzy membership function. These two methods are not quite equivalent. Suppose we provide the weights of ranks as in the third column of the table with 
the reasons we provided above. If we scale them to the range $[0,1]$ and refer to them as a fuzzy membership function, this membership function would not be a reasonable representation of the rank descriptions, because, for example, no one will decide on the membership value of 0.01 for the rank 4 . That is not a simple matter of proportion. On the other hand, given the fuzzy memberships in column 4, for each instructor who prefers to teach his/her number 1 rank course, or for individuals who would like to maximize the overall value of ranks to benefit having more courses run by the right instructors, some skeptical instructors would not use gradation weights to measure the ranks 2 and 3 as 8,000 and 7,000 , respectively, since they are so close to each other and quite close to the highest value of 10,000. Therefore we could not use a simple proportion to transform the membership value of 0.8 (or 0.7) to the scale from 0 to 10,000 .

In some special cases, if a decision maker can design a method or process to collect each of individual's preference values in a way that the collected data have probability interval or random set property, then we may apply the algorithms presented in $[8,9]$ to convert the information to a corresponding membership function.

\section{Teaching Course Assignment Problem Formulation}

This section focuses on an objective function and standard restrictions of the teaching course assignment problem based on the preference data collected in the Department of Mathematics and Computer Science, Chulalongkorn University.

Apart from the preference ranking, each instructor also needs to provide the amount of teaching workload he/she would like to teach. The department counts all seminar levels, senior project, master thesis, and Ph.D. dissertation advising as a part of the teaching duty. Each ith instructor knows his/her load (say $d_{i}$ units) before providing her demand for teaching workload. Out of the total workload duty of 35 units, each person needs to teach at least 18 units and devote $20 \%$ (7 units) for academic community service, or duties assigned by the department/faculty/university. The 18 units teaching workload guarantees that instructors would spend at least half of their total workload for teaching. The maximum teaching allowance is $70 \%$ (24.5 units). The rest will be the research workload. Therefore, instructors have some flexibility in terms of teaching and researching.

The department had the actual of 98 subjects. Some subjects require more than one section. In the case of a subject with only one section but requiring two instructors, it is considered to be two subsections with a half size workload for each subsection. The data set contains the total of $m=120$ sections/subsections and $n=58$ instructors.

In order to formulate our standard constraints and objective function for the teaching course assignment problem, let us define variables, parameters, and terms used in the model in Section "Notation and Meaning of Variables, Parameters, and Sets."
3.1. Standard Restrictions. The notation in Section "Notation and Meaning of Variables, Parameters, and Sets" is used for formulating the standard restrictions as follows.

(i) Each instructor cannot be assigned more than 3 full sections, which will cover more than $70 \%$ of all workload units:

$$
\sum_{j \in J^{1}} x_{i j}+\sum_{j \in J^{2}} x_{i j} \leq 3, \quad \forall i \in I .
$$

(ii) Only one instructor is required for one section/subsection:

$$
\begin{aligned}
& \sum_{i=1}^{n} x_{i j}=1, \quad \forall j \in J^{1}, \\
& \sum_{i=1}^{n} x_{i j}=\frac{1}{2}, \quad \forall j \in J^{2} .
\end{aligned}
$$

(iii) The department avoids assigning multiple teaching sections/subsections of the same subject to one instructor. This restriction prevents an unequal number of preparations per instructor:

$$
\begin{gathered}
\sum_{j \in J_{k} \cap J^{1}} x_{i j} \leq 1, \\
\sum_{j \in J_{k} \cap J^{2}} x_{i j} \leq \frac{1}{2},
\end{gathered}
$$

$\forall i \in I, k \in K$

(iv) Every instructor should meet his/her own requested amount of workload. If not, an excessive workload or a remaining amount of workload would need to be assigned:

$$
\sum_{j=1}^{m} a_{j} x_{i j}-\delta_{i}+\beta_{i}=b_{i}-d_{i}, \quad \forall i \in I .
$$

The set of all feasible solutions to the standard restrictions (1)-(4) is defined as $\Omega$;

$$
\Omega=\{(\mathbf{x}, \boldsymbol{\delta}, \boldsymbol{\beta}) \mid(\mathbf{x}, \boldsymbol{\delta}, \boldsymbol{\beta}) \text { satisfies (1)-(4) }\},
$$

where the bold symbols refer to vectors of corresponding sizes.

3.2. Objective Function Coefficients. As explained in Section 2, we consider two methods for measuring the rank of preference: the weight of rank and the fuzzy number of preference. The $i$ th instructor provides his/her $r_{i}$ th rank of preference to teach the $j$ th section/subsection. The weight of the $r_{i}$ th rank is denoted by $w_{i j}\left(r_{i}\right)$, as follows:

$$
\begin{aligned}
& {\left[w_{i j}(1), w_{i j}(2), w_{i j}(3), w_{i j}(4), w_{i j}(5), w_{i j}(6)\right]} \\
& \quad=[10,000,5,000,500,100,20,0] .
\end{aligned}
$$


In order to compare the weights with the fuzzy memberships, the weights are scaled in proportion to 1 . These scaled weights $c_{i j}\left(r_{i}\right)$ become

$$
\begin{aligned}
& {\left[c_{i j}(1), c_{i j}(2), c_{i j}(3), c_{i j}(4), c_{i j}(5), c_{i j}(6)\right]} \\
& \quad=[1,0.5,0.05,0.01,0.002,0] .
\end{aligned}
$$

The fuzzy preference membership function of the $r_{i}$ th rank is denoted by $\widetilde{c}_{i j}\left(r_{i}\right)$, as follows:

$$
\begin{aligned}
& {\left[\widetilde{c}_{i j}(1), \widetilde{c}_{i j}(2), \widetilde{c}_{i j}(3), \widetilde{c}_{i j}(4), \widetilde{c}_{i j}(5), \widetilde{c}_{i j}(6)\right]} \\
& \quad=[1,0.8,0.7,0.5,0.3,0] .
\end{aligned}
$$

Let $M_{1}$ and $M_{2}$ be penalty terms applied to the excess and deficiency of amount of requested workload, respectively.

Then we can consider a mathematical model of mixedinteger linear programs, (9), for a teaching course assignment problem using the scaled weights of rank and penalty terms in its objective function, satisfying all three goals, (a), (b), and (c), as stated in Section 2, as

$$
\max _{(\mathbf{x}, \boldsymbol{\delta}, \boldsymbol{\beta}) \in \Omega} \sum_{i=1}^{n} \sum_{j=1}^{m} c_{i j} x_{i j}-M_{1} \sum_{i=1}^{n} \delta_{i}-M_{2} \sum_{i=1}^{n} \beta_{i} .
$$

And if we use the fuzzy membership coefficients, $\widetilde{c}_{i j}$, the objective function using the fuzzy teaching preference becomes

$$
\max _{(\mathbf{x}, \boldsymbol{\delta}, \boldsymbol{\beta}) \in \Omega} \sum_{i=1}^{n} \sum_{j=1}^{m} \widetilde{c}_{i j} x_{i j}-M_{1} \sum_{i=1}^{n} \delta_{i}-M_{2} \sum_{i=1}^{n} \beta_{i} .
$$

This is a simple model, certainly less complicated than considering multiobjective model with restrictions (a), (b), and (c). And it lends itself easily to be efficiently reused within a similar environment, after finding the ideal fuzzy membership function, for building the future semester assignments.

Intuitively, the first term of the proposed objective functions should lead to the goals (a) and (b); that is, both models would try to get very few of the lowest preference subjects/sections and get more in the highest preference subjects/sections, at the same time. And the penalty terms satisfy the requirement (c). However, the penalty terms may become the obstacles if some $\delta_{i}$ or $\beta_{i}$ stand out significantly. So $M_{1}$ and $M_{2}$ should be chosen carefully.

Each instructor should be able to fulfill the task he/she promises to do. Therefore, to ensure the promise, the penalized value $M_{2}$ should be much larger than the highest value of the weight of rank/membership value so that the remaining workload will be as small as possible. On the other hand, there should be some rules for the penalty value $M_{1}$. A bit more workload than what instructor requested should be allowed but should not be too much, since it would create unfairness among workers. It seems that a large value of $M_{1}$ forces $\delta_{i}$ term to be smaller in order to maximize the objective function. However, making $\delta_{i}$ too small may not be a good idea, since the model could assign more of some subjects that instructors cannot teach. Instead, choosing $M_{1}$ between
TABLE 2: Optimal course assignment results for problems (9) and (10).

\begin{tabular}{lcc}
\hline \multirow{2}{*}{ Rank } & \multicolumn{2}{c}{ Number of subjects } \\
\hline 1 & Problem (9) & Problem (10) \\
2 & 95 & 91 \\
3 & 9 & 10 \\
4 & 1 & 8 \\
5 & 0 & 0 \\
6 & 1 & 1 \\
\hline
\end{tabular}

the (membership) value of ranks 5 and 6 produces a small amount of the term $M_{1} \sum_{i=1}^{n} \delta_{i}$. Then, for the model to reach optimality, it does not need that many assigned subjects with the least preference to help enhance a higher objective value. This could provide a better teaching quality.

\section{Weight of Rank versus Fuzzy Preference}

With the restriction of penalty terms explained at the end of Section 3, using $\left(M_{1}, M_{2}\right)$ for both problems (9) and (10) as $(0.0015,10)$, the solutions were found using CPLEX version 12.1 and are shown in Table 2.

The results in Table 2 clearly show the advantage of using fuzzy preference over the scaled weights of ranks. Even though the optimal result for problem (9) has more matches for instructors in their preferred sections, it also has more instructors with nonpreferred sections than the result of (10). Furthermore, there should be as few as possible of the nonpreferred sections in order to control the overall department teaching quality. So, comparing these two problems, the results of problem (10) seem more favorable.

4.1. Finding a Better Optimum. Although the solutions to problems (9) and (10) are optimal (under their own models), we do not know, based on these solution alone, if the number of sections with rank 6 is the smallest possible number.

To find this number we suggest the following additional priorities to be added to the standard constraints (1)-(4):

(i) The assignment should contain the smallest number possible of sections with rank 6. Let us define this number as $n_{6}$.

(ii) With $n_{6}$ in hand, the number of sections with rank $5, n_{5}$, should be the next smallest possible, under the standard restrictions.

(iii) With $n_{6}$ and $n_{5}$ in hand, leave the total number of sections with ranks 4, 3, 2, and 1 for the model to arrange/ adjust them for a reasonable optimum objective value.

After we take care of the last two least preferable ranks (ranks 6 and 5) by (i) and (ii), we should get a meaningful better solution that takes care of the workloads and the overall preferences by (iii). 
It seems like we need to handle these constraints (i)-(iii) one at the time. Models (9) or (10) alone cannot interpret the result with these additional priorities. Moreover, the total number of sections in each rank cannot actually be reviewed directly by solving the models, since a solution presents decision variables not the number of sections.

Hence we can follow the following algorithm to obtain a better course assignment than problem (10) using priorities (i)-(iii).

Step 1. Find the smallest number $n_{6}=\sum_{i=1}^{n} \sum_{j=1}^{m} c_{i j}^{(6)} x_{i j}$ as the optimal solution to the following problem:

$$
\min _{(\mathbf{x}, \boldsymbol{\delta}, \boldsymbol{\beta}) \in \Omega} \sum_{i=1}^{n} \sum_{j=1}^{m} c_{i j}^{(6)} x_{i j}
$$

where $\left[c_{i j}^{(6)}(1), c_{i j}^{(6)}(2), c_{i j}^{(6)}(3), c_{i j}^{(6)}(4), c_{i j}^{(6)}(5), c_{i j}^{(6)}(6)\right]$ is $[0,0$, $0,0,0,1]$.

Step 2. Add $n_{6}$ as another restriction to obtain the smallest number $n_{5}=\sum_{i=1}^{n} \sum_{j=1}^{m} c_{i j}^{(5)} x_{i j}$ from

$$
\begin{aligned}
\min _{(\mathbf{x}, \boldsymbol{\delta}, \boldsymbol{\beta}) \in \Omega} & \sum_{i=1}^{n} \sum_{j=1}^{m} c_{i j}^{(5)} x_{i j}, \\
\text { s.t. } & \sum_{i=1}^{n} \sum_{j=1}^{m} c_{i j}^{(6)} x_{i j} \leq n_{6},
\end{aligned}
$$

where $\left[c_{i j}^{(5)}(1), c_{i j}^{(5)}(2), c_{i j}^{(5)}(3), c_{i j}^{(5)}(4), c_{i j}^{(5)}(5), c_{i j}^{(5)}(6)\right]$ is $[0,0$, $0,0,1,0]$.

Step 3. Optimize course assignment to maximize the overall preference and reduce over/underload by solving the problem upon using the preference membership function (8) and $\left(M_{1}, M_{2}\right)=(0.0015,10)$, as follows:

$$
\begin{aligned}
\min _{(\mathbf{x}, \boldsymbol{\delta}, \boldsymbol{\beta}) \in \Omega} & \sum_{i=1}^{n} \sum_{j=1}^{m} \widetilde{c}_{i j} x_{i j}-M_{1} \sum_{i=1}^{n} \delta_{i}-M_{2} \sum_{i=1}^{n} \beta_{i}, \\
\text { s.t. } & \sum_{i=1}^{n} \sum_{j=1}^{m} c_{i j}^{(6)} x_{i j} \leq n_{6}, \\
& \sum_{i=1}^{n} \sum_{j=1}^{m} c_{i j}^{(5)} x_{i j} \leq n_{5} .
\end{aligned}
$$

The solution to those steps was found using CPLEX, giving an optimal course assignment presented in Table 3.

Clearly this is a better solution than that to problem (10).

4.2. Choice of Best Fuzzy Membership Preference. At this point an interesting question arises, whether we could find a fuzzy membership function to solve problem (10) and obtain solution from Table 3 directly, without resorting to Steps 1 to 3 in Section 4.1 by using only one mathematical program
TABLE 3: Optimal course assignment results for problem (13).

\begin{tabular}{lc}
\hline Rank & Number of subjects for problem (13) \\
\hline 1 & 83 \\
2 & 13 \\
3 & 9 \\
4 & 3 \\
5 & 5 \\
6 & 7 \\
\hline
\end{tabular}

without having to verify the values of $n_{6}$ and $n_{5}$ ahead of time. This is more complicated than just an optimization problem with nonlinear objective function (since all $c_{i j}$ 's would also be variable here) because we would have to take care of more than one simple objective. This research has no aim to solve this particular problem directly but, as seen from the solutions of problems (9) and (10), we should be able to improve our fuzzy number of preference so that we can find the optimal solution to system (10) to be like that in Table 3 instead of Table 2.

\section{Improving the Fuzzy Preference}

Let us start with our optimal solution in Table 3 and move backwards to reach a corresponding preference $\widetilde{c}$ using sensitivity analysis. The process proposed below provides an improved fuzzy preference for this particular application.

Step 1. Consider the problem

$$
\begin{aligned}
\min _{(\mathbf{x}, \boldsymbol{\delta}, \boldsymbol{\beta}) \in \Omega} & \sum_{i=1}^{n} \sum_{j=1}^{m} x_{i j}-M_{1} \sum_{i=1}^{n} \delta_{i}-M_{2} \sum_{i=1}^{n} \beta_{i}, \\
\text { s.t. } & \sum_{i=1}^{n} \sum_{j=1}^{m} c_{i j}^{(6)} x_{i j} \leq n_{6}, \\
& \sum_{i=1}^{n} \sum_{j=1}^{m} c_{i j}^{(5)} x_{i j} \leq n_{5}, \\
& \sum_{i=1}^{n} \sum_{j=1}^{m} c_{i j}^{(4)} x_{i j} \leq n_{4}, \\
& \sum_{i=1}^{n} \sum_{j=1}^{m} c_{i j}^{(3)} x_{i j} \leq n_{3}, \\
& \sum_{i=1}^{n} \sum_{j=1}^{m} c_{i j}^{(2)} x_{i j} \leq n_{2}, \\
& \sum_{i=1}^{n} \sum_{j=1}^{m} c_{i j}^{(1)} x_{i j} \leq n_{1},
\end{aligned}
$$


where $\left(M_{1}, M_{2}\right)=(0.0015,10), n_{t}$ is the number of subjects with rank $t$ as stated in Table 3 such that $\sum_{t=1}^{6} n_{t}=120$, and

$$
c_{i j}^{(t)}\left(r_{i}\right)= \begin{cases}1, & \text { when } r_{i}=t \\ 0, & \text { otherwise }\end{cases}
$$

If there is an optimal solution $\hat{x}$ to (14) such that $\sum_{i=1}^{n} \sum_{j=1}^{m} c_{i j}^{(t)} \widehat{x}_{i j}<n_{t}$ for some $t=1,2, \ldots, 6$, then, in order to make $\sum_{i=1}^{n} \sum_{j=1}^{m} \widehat{x}_{i j}=120$ (one instructor is needed for each section/subsection), there must be $t_{1} \neq t$ where $\sum_{i=1}^{n} \sum_{j=1}^{m} c_{i j}^{\left(t_{1}\right)} \widehat{x}_{i j}>n_{t_{1}}$, a contradiction. Hence, the solution from Table 3 is also optimal for (14). Now, consider problem (16) with the original standard constraints by removing all constraints with $n_{t}$ :

$$
\max _{(\mathbf{x}, \boldsymbol{\delta}, \boldsymbol{\beta}) \in \Omega} \sum_{i=1}^{n} \sum_{j=1}^{m} x_{i j}-M_{1} \sum_{i=1}^{n} \delta_{i}-M_{2} \sum_{i=1}^{n} \beta_{i} .
$$

(i) If the solution in Table 3 is optimal for (16), then we can continue the sensitivity analysis in Step 2.

(ii) If the solution is not optimal for (16) we need to check the reduced cost terms respective to the basis matrix of this solution and adjust the objective coefficients of (16) till the associated reduced cost terms are all less than or equal zero. This leads to a new objective function of (16). Continue Step 2 if possible. This case may not be continued if all adjusted objective coefficients are not in between 0 and 1 , since it will not be a fuzzy membership.

Step 2. Use sensitivity analysis to see how small $\tilde{c}_{i j}(6)$ should be by keeping the other $\widetilde{c}_{i j}$ as 1 or the their current value. Change $\widetilde{c}_{i j}^{*}(6)$ in the position of $\widetilde{c}_{i j}(6)$ in the current objective function of (16); we get a new objective function with the same optimal solution. Continue using sensitivity analysis to the new problem to find $\widetilde{c}_{i j}^{*}(5), \ldots, \widetilde{c}_{i j}^{*}(2)$, consecutively in the same fashion. Please keep in mind that we need to pick $\widetilde{c}_{i j}^{*}(6)<\cdots<\widetilde{c}_{i j}^{*}(1)=1$, since they represent fuzzy numbers. We can do so by picking up one value in the range found from sensitivity analysis.

By applying this process we find an ideal fuzzy membership function as

$$
\widetilde{c}_{i j}^{*}\left(r_{i}\right)= \begin{cases}1 ; & \text { if } r_{i}=1, \\ 0.95 ; & \text { if } r_{i}=2, \\ 0.9 ; & \text { if } r_{i}=3, \\ 0.85 ; & \text { if } r_{i}=4, \\ 0.8 ; & \text { if } r_{i}=5, \\ 0 ; & \text { if } r_{i}=6 .\end{cases}
$$

If we use them in model (10) we obtain solution as in Table 3 .

\section{Using Probabilities}

If we decide to interpret the rank of preference of each subject as probability, we need to weight rank 1 the most and then ranks 2, 3, 4, and 5 in the order of its importance, where rank 6 weight is zero, since instructors cannot teach that subject. It is clear that the probability of each rank in different subjects may not be the same when we weight the same way in all subjects. Moreover, the weight we would use for each rank is again "fuzzy." Consider the preference data in Table 4, if, for example, we weight 6 to rank 1 and weight 5 to rank 2 till weight 0 to rank 6 , we will get the probability of each rank of subject "2301101/1" as shown in Table 5, for example, probability of rank 1 for each instructor came from $6 /(6 \cdot 9+$ $5 \cdot 5+4 \cdot 3+3 \cdot 1+2 \cdot 3+0 \cdot 37)=0.06$. After evaluating the probabilities of ranks of all subjects in the similar fashion as one of subject " $2301101 / 1$," we scale them proportionally to 1 and use them as $c_{i j}$ of problem (9). The result of this approach is the same as one for problem (10) in Table 2. It is no surprising that the fuzzy preference in Table 1 could be illustrated using the similar idea (but different weight value), since we can see that the weight has fuzzy nature. However, we still do not know what would be a fuzzy preference to reach the solution as in Table 3 . This shows that this probability approach does not provide a better solution than the fuzzy membership function with coefficients (17).

\section{Conclusion}

Any course assignment problem, where ranking of the choices is available, leads to a fuzzy concept of preference. There is no one universal solution to this type of problems and decision maker needs to choose a suitable fuzzy membership function to be used in each particular problem to find an optimal solution. In this paper we have shown the importance of fuzzy preference and methods for finding and improving the membership function. We have shown that the results using membership function are better than weighing the ranks as well as probability approach.

\section{Notation and Meaning of Variables, Parameters, and Sets}

Set

$I: \quad I=\{1,2,3, \ldots, n\}$ the set of all $n$ instructors

$J: \quad J=\{1,2,3, \ldots, m\}$ the set of all $m$ course sections/subsections

$K: K=\{1,2,3, \ldots, s\}$ the set of all $s$ subjects. Each $k$ th subject contains the total of $\left|J_{k}\right|$ sections/subsections, $k \in K$

$J_{k}: J_{k}=\{j \in J \mid$ the $j$ th section/subsection is a section/subsection of the $k$ th subject $\}$

$J^{1}: J^{1}=\{j \in J \mid$ the $j$ th section requires only one instructor $\}$

$J^{2}: J^{2}=\{j \in J \mid$ the $j$ th subsection requires two instructors $\}$ $J^{1} \cap J^{2}=\emptyset$ and $J^{1} \cup J^{2}=J$. 


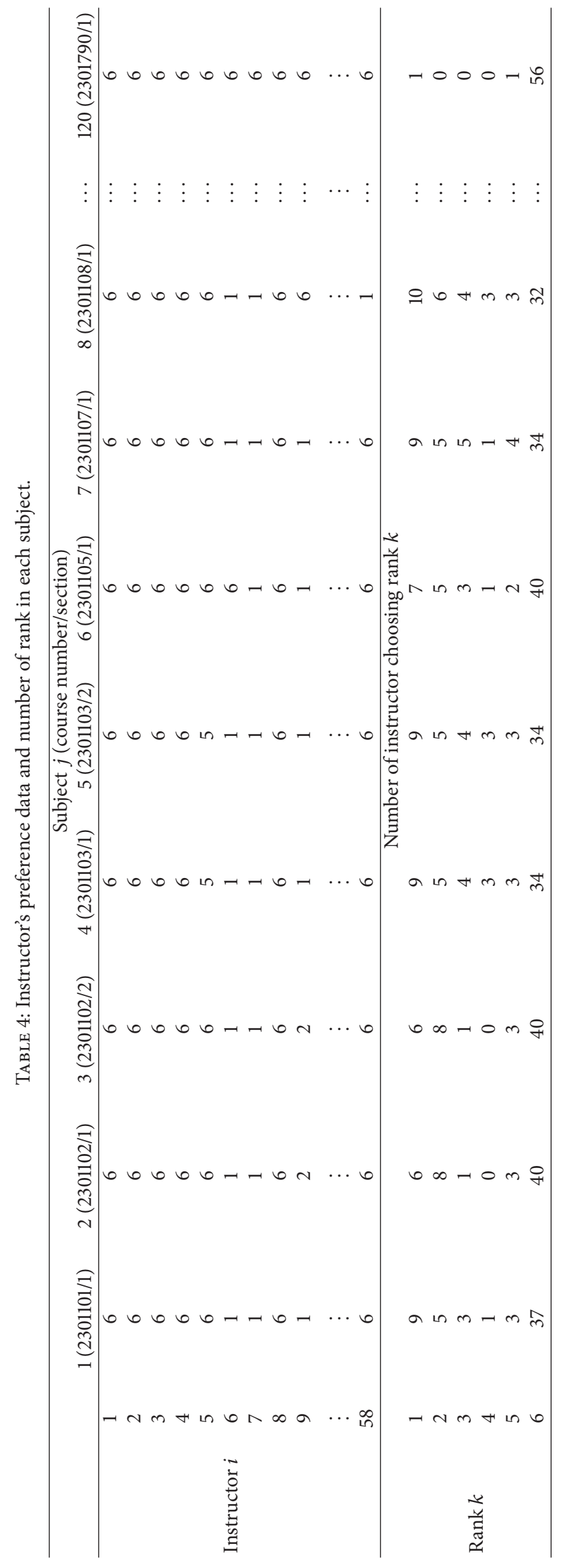


TABLE 5: Probability of each rank for the course number "2301101" Section 1.

\begin{tabular}{lcccc}
\hline Rank $k$ & Weight & $\begin{array}{c}\text { Number of } \\
\text { instructors }\end{array}$ & $\begin{array}{c}\text { Probability for } \\
\text { each instructor }\end{array}$ & $\begin{array}{c}\text { Proportion } \\
\text { to 1 }\end{array}$ \\
\hline 1 & 6 & 9 & 0.06 & 1 \\
2 & 5 & 5 & 0.05 & 0.83 \\
3 & 4 & 3 & 0.04 & 0.67 \\
4 & 3 & 1 & 0.03 & 0.5 \\
5 & 2 & 3 & 0.02 & 0.33 \\
6 & 0 & 37 & 0 & 0 \\
\hline
\end{tabular}

Parameter

$a_{j}$ : the amount of workload earned by teaching the $j$ th section/subsection

$b_{i}$ : the $i$ th instructor's requested workload

$c_{i j}$ : the scaled weight of rank of the $j$ th section/subsection with respect to the $i$ th instructor

$\widetilde{c}_{i j}:$ the fuzzy membership value of preference of the $i$ th instructor to the $j$ th section/subsection

$d_{i}$ : the seminar, project and thesis advisor duties of the $i$ th instructor. The $i$ th instructor needs to fulfill the amount of teaching workload of $b_{i}-d_{i}$.

\section{Variable}

$x_{i j}: x_{i j}=1$, the $i$ th instructor teaches the $j$ th section, $j \in J^{1} ; 1 / 2$, the $i$ th instructor teaches the $j$ th subsection, $j \in J^{2} ; 0$, otherwise

$\delta_{i}: \delta_{i} \geq 0$ an extra workload that the $i$ th instructor has on top of the requested workload

$\beta_{i}: \beta_{i} \geq 0$ a remaining amount of the requested workload after deducting from the actual assigned workload.

\section{Conflict of Interests}

The authors declare that there is no conflict of interests regarding the publication of this paper.

\section{Acknowledgments}

The authors would like to thank the Department of Mathematics and Computer Science, Faculty of Science, Chulalongkorn University, for the data used in this paper. Special thanks are due to Miss Rujira Wisuttirattanamanee for the solutions of problems (10) and (13). They would also like to thank the anonymous reviewers, for their valuable comments, hints, and suggestions that helped to improve the paper.

\section{References}

[1] R. Wisuttirattanamanee, Solving the teacher assignment problem with $\alpha$-level from instructors' fuzzy satisfaction [M.S. thesis], Chulalongkorn University, Bangkok, Thailand, 2013.

[2] P. Thipwiwatpotjana, "Course assignment problem with interval requested workload," in Proceedings of the IEEE Conference on Norbert Wiener in the 21st Century, pp. 1-5, Boston, Mass, USA, June 2014.

[3] Y. Duan, Y.-B. Zhong, and Y.-Q. Li, "Application research on FSDM-based GA in optimizing curriculum schedule model in universities," Fuzzy Information and Engineering, vol. 4, no. 2, pp. 217-228, 2012.

[4] N. A. Ismayilova, M. Sagir, and R. N. Gasimov, "A multiobjective faculty-course-time slot assignment problem with preferences," Mathematical and Computer Modelling, vol. 46, no. 7-8, pp. 1017-1029, 2007.

[5] A. Chaudhuri and K. De, "Fuzzy genetic heuristic for university course timetable problem," International Journal of Advances in Soft Computing and Its Applications, vol. 2, no. 1, pp. 100-123, 2010.

[6] R. Perzina and J. Ramik, "Self-learning genetic algorithm for a timetabling problem with fuzzy constraints," International Journal of Innovative Computing, Information and Control, vol. 9, no. 11, pp. 4565-4582, 2013.

[7] G. J. Klir, "Is there more to uncertainty than some probability theorists might have us believe?" International Journal of General Systems, vol. 15, no. 4, pp. 347-378, 1989.

[8] P. Boodgumarn, P. Thipwiwatpotjana, and W. A. Lodwick, "When a probability interval is a random set," ScienceAsia, vol. 39, no. 3, pp. 319-326, 2013.

[9] P. Thipwiwatpotjana and W. A. Lodwick, "A relationship between probability interval and random sets and its application to linear optimization with uncertainties," Fuzzy Sets and Systems, vol. 231, pp. 45-57, 2013. 


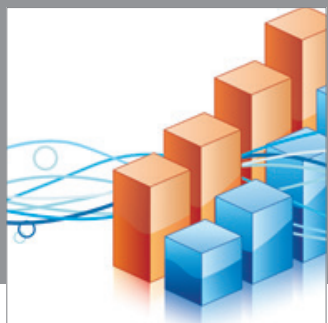

Advances in

Operations Research

mansans

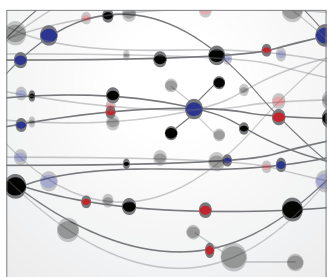

The Scientific World Journal
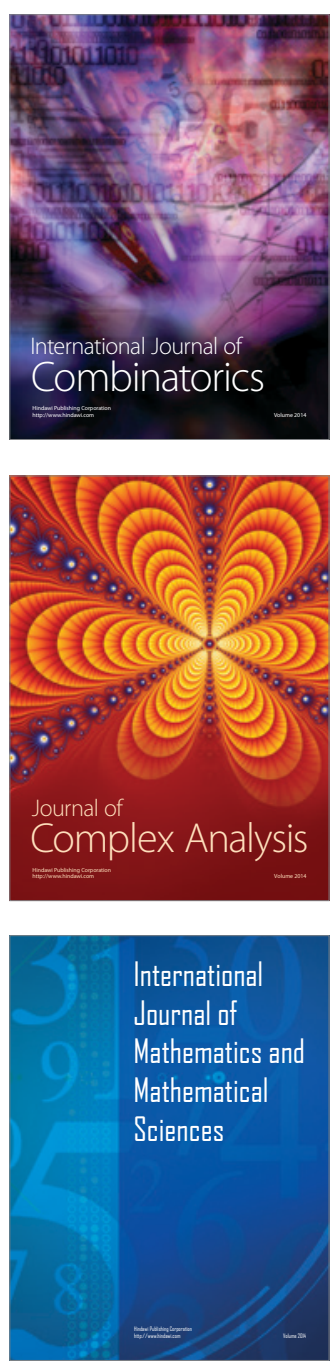
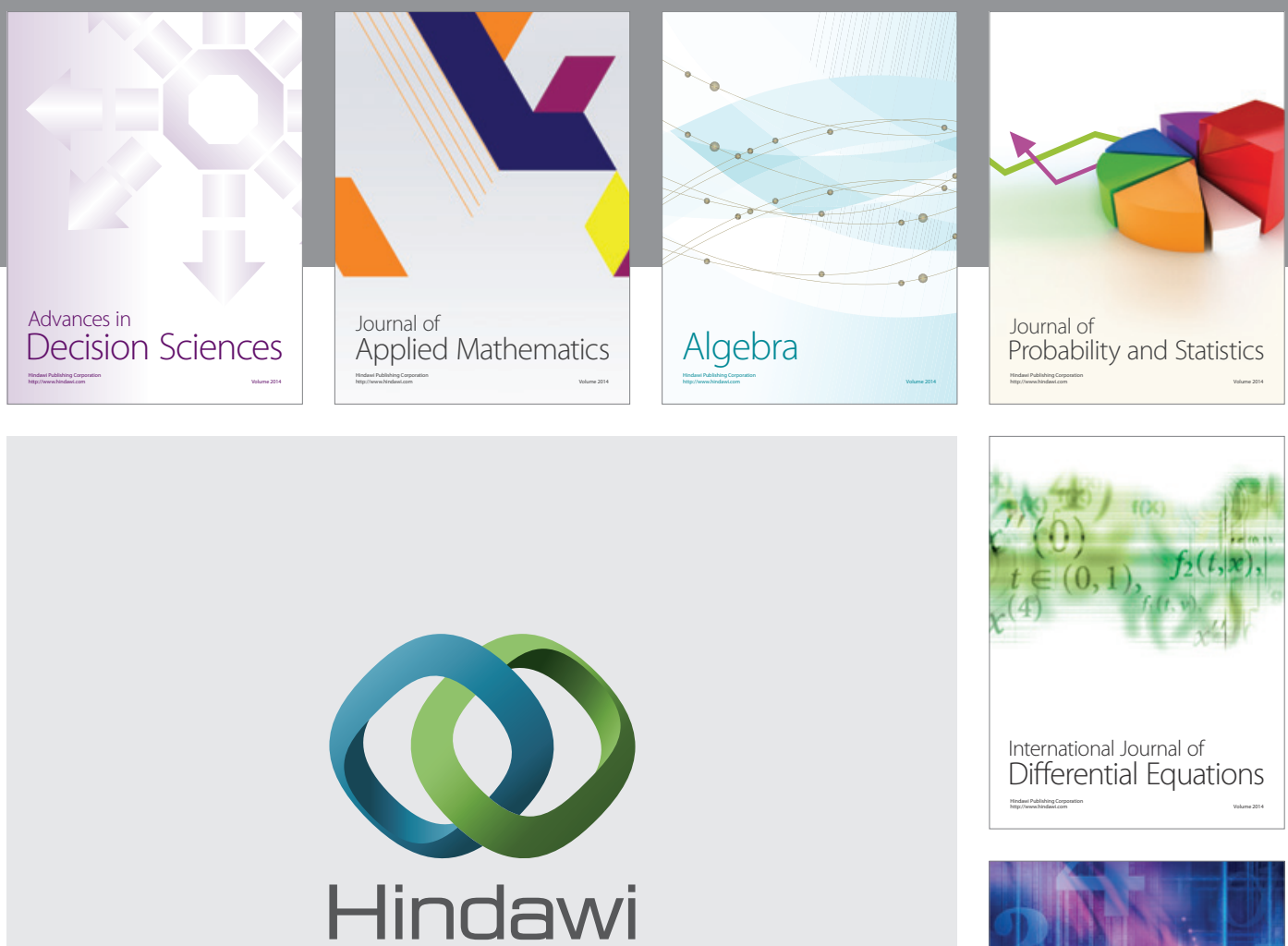

Submit your manuscripts at http://www.hindawi.com
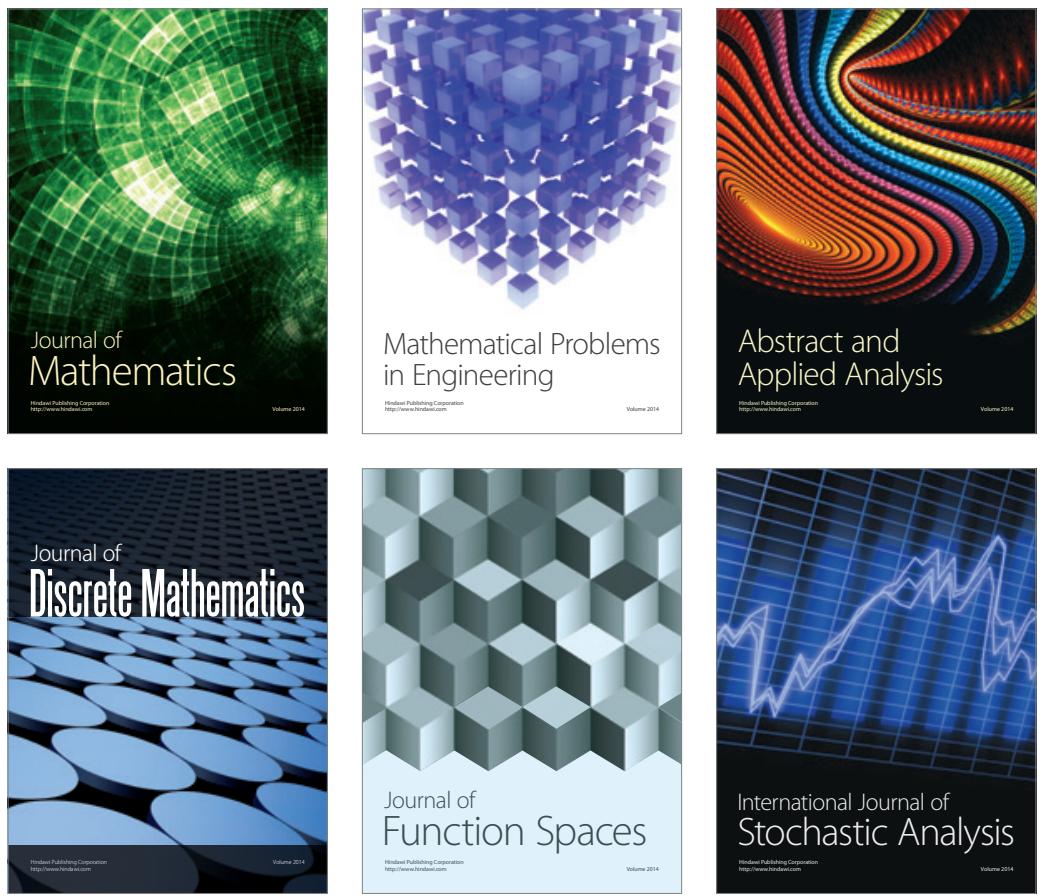

Journal of

Function Spaces

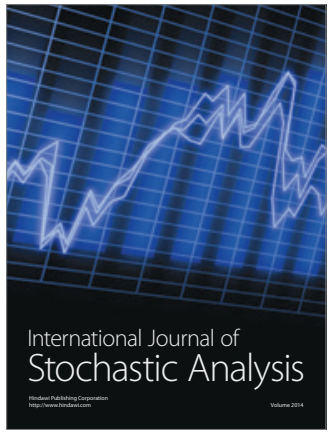

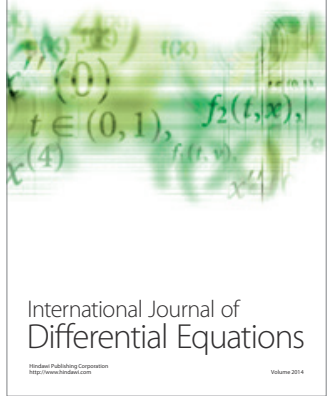
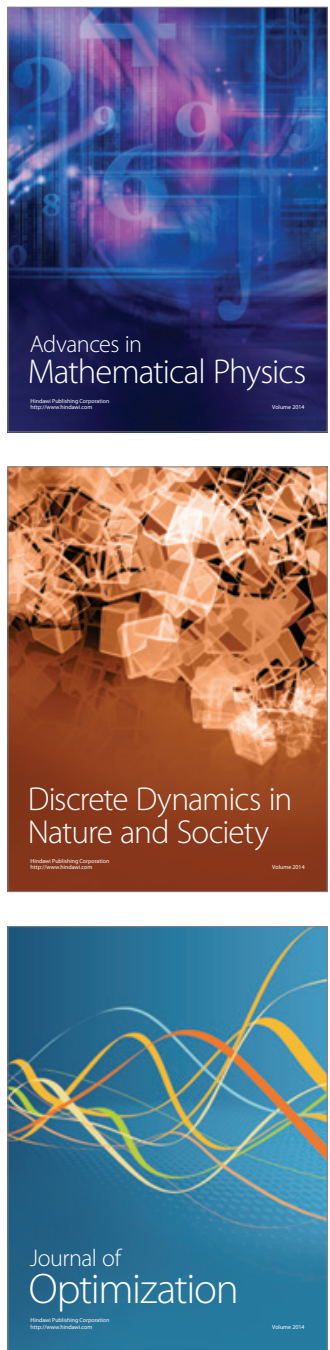